\title{
Reduced-order disturbance observer based adaptive reaching law control for discretized MIMO systems with unmatched uncertainties
}

Cong Wang ( $\sim$ 18B904036@stu.hit.edu.cn )

Harbin Institute of Technology https://orcid.org/0000-0003-1646-5104

Hongwei Xia

Harbin Institute of Technology

Yanmin Wang

Harbin Institute of Technology

Shunqing Ren

Harbin Institute of Technology

\section{Research Article}

Keywords: Discrete-time sliding mode control, Adaptive reaching law, Reduced-order disturbance observer, Unmatched disturbance, Multi-input multi-output system

Posted Date: June 14th, 2021

DOI: https://doi.org/10.21203/rs.3.rs-168729/v1

License: (9) This work is licensed under a Creative Commons Attribution 4.0 International License. Read Full License 


\title{
Reduced-order disturbance observer based adaptive reaching law control for discretized MIMO systems with unmatched uncertainties
}

\author{
Cong Wang · Hongwei Xia · Yanmin Wang · Shunqing Ren
}

Received: date / Accepted: date

\begin{abstract}
In this paper, an adaptive discrete-time sliding mode control based on reduced-order disturbance observer is proposed for discretized multi-input multi-output systems subjected to unmatching condition. By using the designed discrete reduced-order disturbance observer, a new sliding surface is constructed to counteract the unmatched uncertainties. Then, to guarantee a smaller width of the quasi sliding mode domain, an adaptive reaching law is developed, whose switching gain is adaptively tuned to prevent overestimation of disturbance on the premise of ensuring the reaching condition of sliding surface; meanwhile, the ranges of the quasi sliding mode band and attractiveness region are deduced. The proposed control algorithm has low computational complexity and needs no information about the upper bound of unmatched disturbance. The simulations on the control of a bank-to-turn missile demonstrate that the proposed method can effectively reject unmatched disturbance, and provide higher accuracy in comparison with traditional methods.
\end{abstract}

Keywords Discrete-time sliding mode control · Adaptive reaching law $\cdot$ Reduced-order disturbance observer .

Unmatched disturbance $\cdot$ Multi-input multi-output system

\section{Introduction}

As an effective robust control technology, sliding mode control (SMC) has been widely investigated in theoretical research and extensively adopted in industrial application with advantages such as simplicity in implementation, guaranteed stability, and robustness against parameter variations

C. Wang $(\bowtie) \cdot H$. Xia $\cdot$ S. Ren

School of Astronautics, Harbin Institute of Technology, Harbin, China

E-mail: 18B904036@ stu.hit.edu.cn

Y. Wang

School of Automation and Electrical Engineering, Harbin Institute of Technology, Harbin, China and external disturbances $[26,10]$. Despite the aforementioned advantages, it has a major drawback in the application of SMC, i.e., it can only reject the uncertainties meeting the so-called matching condition, which means that only when the uncertainties are in the same channel of the control input, can the controller eliminate the adverse effect of disturbances $[16,35]$. Inevitably, the lumped disturbances including parameter uncertainties, external disturbances and unmodeled dynamics in practical systems may not satisfy the matching condition [20]. Concerning this problem, a lot of methods have been studied, such as linear matrix inequality (LMI) based approach [6], integral sliding mode control [3], extended state observer based control [29], disturbance observer based control (DOBC) [5, 18].

Among the methods mentioned above, DOBC has drawn a great attention in control society in the past few years as it effectively compensates the undesirable effects of unknown disturbances without damaging its nominal control performance [4,22]. For example, in the work of Yang et al [33], the DOBC has been developed in the SMC approach for single input single output systems with unmatched uncertainties. In order to reduce the computational load of digital signal processors, a reduced-order disturbance observer (RODO) was studied by Kim et al [15], in which unknown inputs and states were estimated instead of the whole states estimation. Based on virtual signal control technique, a fullorder terminal sliding mode (TSM) control was proposed to completely reject unmatched uncertainties and to force the output of multi-input multi-output (MIMO) systems to converge to equilibrium point in [9]. However, these methods are only available for continuous-time systems.

With the rapid development of digital processors in control systems, a considerable amount of researches on discretetime sliding mode control (DSMC) have been published [28, 32]. For DSMC systems, the system trajectories are not able to slide on the surface but within a band called quasi sliding 
mode (QSM) band owing to finite sampling frequency, resulting in that many excellent characteristics in continuoustime domain no longer exist, such as invariable property for disturbances [2]. Among the existing methods on DSMC, the discrete reaching law proposed by Gao [11] is often utilized because of simple implementation, which directly establishes a reaching condition of sliding surface, and specifies the dynamic characteristics of systems during the reaching phase. The width of the QSM band is determined by parameters in reaching law and the spread of disturbances, and thus chattering phenomenon can be reduced by proper design and accurate disturbance compensation [13]. Zhang et al have proposed a new reaching law containing a nonsmooth term and a second order difference of disturbance to reduce the range of QSM band and attenuate chattering by using a novel disturbance observer [36]. A new adaptive reaching law adopting a negative power term that contains the absolute value of sliding variable has been conducted in [17], and the control strategy ensures a small width of QSM band without jeopardizing the reaching time of sliding surface. But these approaches are unable to reject unmatched disturbances.

For unmatched disturbances in discrete-time domain, a lot of elegant results have been provided for DSMC systems [19]. In [34], an model-free robust controller has been designed for discrete-time systems with bounded unmatched disturbances, and it is suitable for off-line control systems. Within the LMI framework [31], the state variables of uncertain systems were guaranteed to converge into a residual set of the origin by an adaptive controller, but the uncertainties must be assumed to be norm-bounded. Based on the delay estimation technique, the discrete reaching law was constructed for discrete-time dynamic systems with unmatched disturbances [7], and the QSM band of sliding surface is in the order of $O(h)$ where $h$ is sampling time and $O(h)$ denotes the item with orders higher than or equal to $h$. Utilizing a weighted moving average method, Sharma and Janardhanan developed a discrete-time higher-order SMC scheme for single input linear time invariant systems in the presence of unmatched uncertainties [21]. On the basis of the reciprocally convex approach and Lyapunov-Krasovskii technique [25], the unmatched disturbance problem for a class of discretetime systems has been dealt with very well, but high computational complexity is its inherent defect. In [30], based on sliding mode disturbance observer, a recursive TSM controller has been studied to reject measurement noises and external disturbances. In [14], an adaptive TSM control was designed for nonlinear systems subjected to disturbances, in which it was not necessary to know the upper bound of disturbance. Another newly popular DSMC, discrete-time integral sliding mode control (DISMC), has been reported to be an efficient scheme to reject unmatched uncertainties due to its simplicity and robustness [1,27], but it also brings some side effects such as large overshoot and nominal control performance degradation.

Noting that the advantages of reaching law approach lie in its good performances such as chattering attenuation and simple realization, we will propose a RODO based adaptive reaching law control for discretized MIMO systems subjected to unmatched disturbances. To be specific, the major contributions are listed as:

- A discrete RODO is introduced, which can accurately estimate unmatched disturbances by using the state information at current and previous moments.

- A new discrete sliding surface with disturbance compensation is designed to counteract the unmatched disturbances.

- The reaching law with adaptive switching gain is established to reduce the wide of QSM band, and the ranges of the QSM band and attractiveness region are obtained.

- Based on the proposed RODO and adaptive reaching law, the sliding mode controller is developed, and the active disturbance rejection can be achieved.

This paper is organized as follows. The system and problem descriptions are formulated in Section 2. In Section 3, reduced-order disturbance observer, sliding surface, adaptive reaching law and discrete sliding mode controller are designed respectively. The simulations are presented in Section 4 to validate this paper.

\section{System and problem descriptions}

Consider a discrete-time MIMO system with disturbance:

$\bar{x}(k+1)=\bar{\Phi} \bar{x}(k)+\bar{\Gamma} u(k)+\bar{d}(k)$,

where $\bar{x}(k) \in R^{n}, u(k) \in R^{m}$, and $\bar{d}(k) \in R^{n}$ are the system state, the control input, and the external disturbance respectively; $(\bar{\Phi}, \bar{\Gamma})$ is a controllable pair; $\bar{\Gamma}=\left[\bar{\Gamma}_{1}, \bar{\Gamma}_{2}\right]^{\mathrm{T}}$, $\bar{\Gamma}_{1} \in R^{(n-m) \times m}, \bar{\Gamma}_{2} \in R^{m \times m}$ is nonsingular.

Definition 1 For the disturbance vector $\bar{d}(k)$, its components in the same channel as the control input (or by state transformation), are called matched disturbances, whereas the rests are considered to be unmatched disturbances.

By state transformation

$x(k)=T \bar{x}(k), T=\left[\begin{array}{cc}I_{n-m} & -\bar{\Gamma}_{1} \bar{\Gamma}_{2}^{-1} \\ 0 & \bar{\Gamma}_{2}^{-1}\end{array}\right]$,

the system (1) can be transformed as:

$\left\{\begin{aligned} x_{1}(k+1) & =\Phi_{11} x_{1}(k)+\Phi_{12} x_{2}(k)+d_{u}(k), \\ x_{2}(k+1) & =\Phi_{21} x_{1}(k)+\Phi_{22} x_{2}(k)+u(k)+d_{m}(k), \\ y(k) & =x_{1}(k),\end{aligned}\right.$ 
where $I_{n-m}$ is an identity matrix; $x(k)=\left[x_{1}^{\mathrm{T}}(k), x_{2}^{\mathrm{T}}(k)\right], x_{1}(k) \in$ $R^{n-m}, x_{2}(k) \in R^{m} ; y(k) \in R^{n-m}$ is the system output; $d_{m}(k)=$ $\left[d_{n-m+1}(k), d_{n-m+2}(k), \ldots, d_{n}(k)\right]^{\mathrm{T}}$ and $d_{u}(k)=\left[d_{1}(k), d_{2}(k)\right.$, $\left.\ldots, d_{n-m}(k)\right]^{\mathrm{T}}$ represent matched and unmatched disturbances respectively. Referring to [1], we can get that $d_{m}(k)=O(h)$ and $d_{u}(k)-d_{u}(k-1)=O\left(h^{2}\right)$. Define $\varepsilon_{i}=\sup _{k \geq 0}\left(\mid d_{i}(k)-\right.$ $\left.d_{i}(k-1) \mid\right), i=1, \ldots, n-m$, denoted as $\varepsilon=\left[\varepsilon_{1}, \varepsilon_{2}, \ldots, \varepsilon_{n-m}\right]^{\mathrm{T}}$, and $k_{j}=\sup _{k \geq 0}\left|d_{j}(k)\right|, j=n-m+1, \ldots, n$, which are expressed as a matrix form $K_{2}=\left[k_{n-m+1}, k_{n-m+2}, \ldots, k_{n}\right]^{\mathrm{T}}$. Then we can conclude that $\varepsilon=O\left(h^{2}\right)$ and $K_{2}=O(h)$, and thus the unmatched disturbance is unnecessary to be bounded.

For system (3), the general linear sliding mode (LSM) surface is defined as

$\bar{s}(k)=\bar{C}_{1} x_{1}(k)+\bar{C}_{2} x_{2}(k)$,

where $\bar{C}_{1} \in R^{m \times(n-m)}, \bar{C}_{2} \in R^{m \times m}$ is nonsingular. According to discrete equivalent control [23], it has $\bar{s}(k+1)=0$, and then (4) is changed as

$x_{2}(k)=-\bar{C}_{2}^{-1} \bar{C}_{1} x_{1}(k)$.

Substituting (5) into (3) yields the reduced-order system

$x_{1}(k+1)=\left(\Phi_{11}-\Phi_{12} \bar{C}_{2}^{-1} \bar{C}_{1}\right) x_{1}(k)+d_{u}(k)$.

The matrices $\bar{C}_{1}$ and $\bar{C}_{2}$ can be designed to ensure the convergence of system (6), but the unmatched disturbance $d_{u}(k)$ will deteriorate the performance of the discrete system. Specifically, the system states are unable to converge to the desired equilibrium point even though they are forced to reach the sliding surface, leading to the appearance of limit cycles. This is the reason why traditional DSMC is sensitive to unmatched disturbances.

\section{Main results}

\subsection{Reduced-order disturbance observer}

In order to counteract the unmatched disturbance in (6), first of all, it should be able to be estimated by the current and previous states. We design a discrete RODO, which can estimate $d_{u}(k)$ in real time, as follows:

$$
\left\{\begin{aligned}
r(k)= & L x_{1}(k), L=\operatorname{diag}\left\{\lambda_{1}, \lambda_{2}, . ., \lambda_{n-m}\right\}, \\
z(k+1)= & \left(I_{n-m}-L\right) z(k)-L\left[r(k)+\Phi_{12} x_{2}(k)\right. \\
& \left.+\left(\Phi_{11}-I_{n-m}\right) x_{1}(k)\right], \\
\hat{d}_{u}(k+1)= & z(k+1)+r(k+1),
\end{aligned}\right.
$$

where $0<\lambda_{1}, \ldots, \lambda_{n-m}<2, \hat{d}_{u}(k) \in R^{n-m}$ is the disturbance estimation, $z(k) \in R^{n-m}$ and $r(k) \in R^{n-m}$ are the internal states of disturbance observer, and $L$ is the observer gain matrix to be designed. Defining disturbance estimation error $e(k)=d_{u}(k)-\hat{d}_{u}(k)$, the convergence accuracy of $\hat{d}_{u}(k)$ is given in the following theorem.
Theorem 1 The discrete reduced-order disturbance observer (7) is asymptotically stable and the estimation error $e(k)=$ $\left[e_{1}(k), e_{2}(k), \ldots, e_{n-m}(k)\right]^{\mathrm{T}}$ will exponentially approach the following boundary layer:

$\Psi \triangleq\left\{e_{i}(k)|| e_{i}(k) \mid \leq \frac{\varepsilon_{i}}{\lambda_{i}}, i=1, \ldots, n-m\right\}$

Proof It follows from (7) that

$$
\begin{aligned}
\hat{d}_{u}(k+1)= & \left(I_{n-m}-L\right) z(k)-L\left[r(k)+\Phi_{12} x_{2}(k)\right. \\
& \left.+\left(\Phi_{11}-I_{n-m}\right) x_{1}(k)\right]+r(k+1) \\
= & z(k)+L x_{1}(k)-L z(k)-L r(k)+L x_{1}(k+1) \\
& -L \Phi_{11} x_{1}(k)-L \Phi_{12} x_{2}(k) \\
= & \hat{d}_{u}(k)-L \hat{d}_{u}(k)+L d_{u}(k) .
\end{aligned}
$$

From (9), it can be derived that

$$
\begin{aligned}
e(k+1)-e(k) & =d_{u}(k+1)-\hat{d}_{u}(k+1)-\left(d_{u}(k)-\hat{d}_{u}(k)\right) \\
& =d_{u}(k+1)-d_{u}(k)-\left(\hat{d_{u}}(k+1)-\hat{d}_{u}(k)\right) \\
& =-L\left(d_{u}(k)-\hat{d}_{u}(k)\right)+d_{u}(k+1)-d_{u}(k) \\
& =-L e(k)+d_{u}(k+1)-d_{u}(k) .
\end{aligned}
$$

Then formula $e(k+1)=\left(I_{n-m}-L\right) e(k)+d_{u}(k+1)-d_{u}(k)$ can be obtained, which implies that

$$
\begin{aligned}
\left|e_{i}(k)\right| & =\left|\left(1-\lambda_{i}\right) e_{i}(k-1)+d_{i}(k)-d_{i}(k-1)\right| \\
& =\left|\left(1-\lambda_{i}\right)^{k} e_{i}(0)+\sum_{j=1}^{k}\left(1-\lambda_{i}\right)^{k-j}\left(d_{i}(j)-d_{i}(j-1)\right)\right| \\
& \leq\left|1-\lambda_{i}\right|^{k}\left|e_{i}(0)\right|+\left|\sum_{j=1}^{k}\left(1-\lambda_{i}\right)^{k-j}\right| d_{i}(j)-d_{i}(j-1)|| \\
& \leq\left|1-\lambda_{i}\right|^{k}\left|e_{i}(0)\right|+\left|\sum_{j=1}^{k}\left(1-\lambda_{i}\right)^{k-j} \varepsilon_{i}\right| \\
& =\left|1-\lambda_{i}\right|^{k}\left|e_{i}(0)\right|+\frac{\varepsilon_{i}\left|1-\left(1-\lambda_{i}\right)^{k}\right|}{\lambda_{i}}
\end{aligned}
$$

Since $-1<1-\lambda_{i}<1, i=1, \ldots, n-m$, then $\left|\left(1-\lambda_{i}\right)^{k}\right|=$ $\left|1-\lambda_{i}\right|^{k} \rightarrow 0$ with $k \rightarrow \infty$ such that the upper bound of $e(k)$ can be deduced as $\left|e_{i}(k)\right| \leq \varepsilon_{i} / \lambda_{i}=O\left(h^{2}\right)$ in steady state. Thus, the discrete RODO (7) is asymptotically stable, and the disturbance estimation error will converge to the region $\Psi$ in (8) at exponential rate.

As shown in (7), $\hat{d}_{u}(k+1)$ consists of $z(k+1)$ and $x_{1}(k+$ $1)$, and thus at the beginning of the RODO operation, the initial value $z(0)$ needs to be determined. Here the initial state of disturbance observer is set as $\hat{d}_{u}(0)=0$, and then the initialization of internal states of the RODO is as follows: $r(0)=L x_{1}(0), z(0)=\hat{d}_{u}(0)-r(0)=-L x_{1}(0)$. In addition, if the unmatched disturbance $d_{u}(k)$ is slowly time-varying, i.e., $d_{u}(k+1)-d_{u}(k) \rightarrow 0$ as $k \rightarrow \infty$, then it follows from (8) that $|e(k)| \rightarrow 0$ and the RODO asymptotically converges to zero, which is a special case of the unmatched disturbance. 
Remark 1 In the design of full-order disturbance observers [12], the whole system structure must be known including the input $u(k)$ and state matrix $\left(\Phi_{11}, \Phi_{12}, \Phi_{21}\right.$ and $\left.\Phi_{22}\right)$. But for the proposed RODO, only partial system information, $\Phi_{11}$ and $\Phi_{12}$, are required. Accordingly, the RODO in this paper needs less system occupation and is easier to implement in real practice. At the same time, due to the reduction of dimension, the RODO takes up less computing resources, which guarantees the practicability of the observer.

Remark 2 In the $\operatorname{RODO}(7)$, if $\lambda_{i}=1, i=1, \ldots, n-m$, then it follows from (9) that $\hat{d}_{u}(k+1)=d_{u}(k)$, which is the form of delay disturbance estimator that has been investigated in many literatures $[23,17]$. Therefore, the proposed RODO can be viewed as a generalization of delay disturbance estimator. As a matter of fact, the parameters $\lambda_{i}, i=1, \ldots, n-m$, can be designed to be greater than 1 to reduce the disturbance estimation error, and we can also relieve chattering phenomenon by choosing relatively small values of $\lambda_{i}$. The RODO improves the degrees of design freedom of delay disturbance estimator for customized system performances.

\subsection{Sliding surface design}

From the analysis of (6), we know that the general LSM surface cannot eliminate the deleterious effect of unmatched disturbances. Based on the RODO depicted in (7), a new sliding surface containing disturbance estimation for system (1) is designed as

$s(k)=C_{1} x_{1}(k)+C_{2} x_{2}(k)+G \hat{d}_{u}(k)$,

where $s(k)=\left[s_{1}(k), s_{2}(k), \ldots, s_{m}(k)\right]^{\mathrm{T}} ; C_{1} \in R^{m \times(n-m)}, C_{2} \in$ $R^{m \times m}$ and $G \in R^{m \times(n-m)}$ are constant parameter matrices to be designed, and $C_{2}$ is nonsingular; $\hat{d}_{u}(k)$ is the disturbance estimation provided by (7). Assuming that discrete sliding surface $s(k)=0$ can be reached, the sliding mode motion can be described by

$x_{2}(k)=-C_{2}^{-1} C_{1} x_{1}(k)-C_{2}^{-1} G \hat{d}_{u}(k)$.

Combining (3) and (13), the dynamic equation of $x_{1}(k)$ can be generated as

$$
\begin{aligned}
x_{1}(k+1)= & \left(\Phi_{11}-\Phi_{12} C_{2}^{-1} C_{1}\right) x_{1}(k) \\
& -\Phi_{12} C_{2}^{-1} G \hat{d}_{u}(k)+d_{u}(k) .
\end{aligned}
$$

Substituting $d_{u}(k)=\hat{d}_{u}(k)+e(k)$ into (14) gives

$$
\begin{aligned}
x_{1}(k+1)= & \left(\Phi_{11}-\Phi_{12} C_{2}^{-1} C_{1}\right) x_{1}(k) \\
& +\left(I_{n-m}-\Phi_{12} C_{2}^{-1} G\right) \hat{d}_{u}(k)+e(k) .
\end{aligned}
$$

It can be seen from (15) that there are three parts in the expression of $x_{1}(k+1)$. They will be discussed one by one. The pole assignment method is adopted for the term $\left(\Phi_{11}-\right.$
$\left.\Phi_{12} C_{2}^{-1} C_{1}\right) x_{1}(k)$, i.e., $C_{1}$ and $C_{2}$ need to be designed such that all the eigenvalues of matrix $\left(\Phi_{11}-\Phi_{12} C_{2}^{-1} C_{1}\right)$ lie within the unit disk. It is obvious that designing $n-m$ eigenvalues involves $n-m$ parameters and remaining $m n-(n-m)$ parameters of $C_{1}$ and $C_{2}$ are free. Furthermore, consider $m n-$ $(n-m)-m^{2}=(m-1)(n-m) \geq 0$, which leaves one degree of freedom in the design of $C_{2}$. For the convenience of design and realization, we choose $C_{2}=h I_{m}$ subsequently in this paper. From (8), we can infer that the estimation error satisfies $|e(k)| \leq O\left(h^{2}\right)$ as iterative number $k$ increases. For the disturbance term $\left(I_{n-m}-\Phi_{12} C_{2}^{-1} G\right) \hat{d}_{u}(k)$, the parameter matrix $G$ should be designed to make $\left(I_{n-m}-\Phi_{12} C_{2}^{-1} G\right)=$ 0 true so that $\hat{d}_{u}(k)$ is completely eliminated. Then it has $\Phi_{12} C_{2}^{-1} G=I_{n-m}$, i.e., $G$ is right pseudo-inverse of $\Phi_{12} C_{2}^{-1}$. It should be noted that only when $\Phi_{12}$ is row full rank, i.e., $\operatorname{Rank}\left(\Phi_{12}\right)=n-m$, does the parameter matrix $G$ exist, where $\operatorname{Rank}(\cdot)$ denotes the rank of a matrix, and it is given as

$G=\left(\Phi_{12} C_{2}^{-1}\right)^{\mathrm{T}}\left[\Phi_{12} C_{2}^{-1}\left(\Phi_{12} C_{2}^{-1}\right)^{\mathrm{T}}\right]^{-1}$

\subsection{Design of adaptive reaching law}

Through the above analysis, we can see that as soon as the sliding surface (12) is reached, the unmatched disturbance can be compensated. To reduce the width of the QSM band for the designed sliding surface (12), a novel adaptive reaching law is proposed as

$$
\begin{aligned}
s(k+1)= & \left(I_{m}-Q_{1} h\right) s(k)-Q_{2}(k) h \operatorname{sgn}(s(k)) \\
& +\left(C_{1}+G L\right)\left(d_{u}(k)-\hat{d}_{u}(k)\right)+C_{2} d_{m}(k),
\end{aligned}
$$

where parameter matrices $Q_{1}=\operatorname{diag}\left\{q_{11}, q_{12}, \ldots, q_{1 m}\right\}$ and $Q_{2}(k)=\operatorname{diag}\left\{q_{21}(k), q_{22}(k), \ldots, q_{2 m}(k)\right\}$ need to be designed; $\operatorname{sgn}(s(k))=\operatorname{diag}\left\{\operatorname{sgn}\left(s_{1}(k)\right), \operatorname{sgn}\left(s_{2}(k)\right), \ldots, \operatorname{sgn}\left(s_{m}(k)\right)\right\}$. In the reaching law (17), it can be found that there are $m$ sliding manifolds for the $m$-input system (1), and each of them contains a single switching function. The adaptive gains of these switching functions are associated with disturbance estimation. To be more specific, the adaptive gain terms $q_{2 i}(k), i=$ $1, \ldots, m$, should be larger than corresponding disturbance terms in reaching law (17) at all times, which implies that

$q_{2 i}(k) h>\left|\left(C_{1}+G L\right)\left(d_{u}(k)-\hat{d}_{u}(k)\right)+C_{2} d_{m}(k)\right|_{i}$,

where $|\cdot|_{i}$ represents the absolute value of the $i$ th element in column vector. The relation in the formula (18) must be satisfied for termed reachability condition of DSMC systems, which means that the system trajectory of (1) is driven towards the sliding surface (12) and then remains on it thereafter. It is easy to give the expression $q_{2 i}(k)=\mid C_{2}^{-1}\left(C_{1} L^{-1}+\right.$ $G) \varepsilon+\left.C_{2} K_{2}\right|_{i}$ that satisfies (18), but it possesses some conservativeness. For the slowly time-varying unmatched disturbance, we will further give an adaptive gain with less conservativeness. In order to facilitate more intuitive derivation, the following definition and lemma are first given. 
Definition 2 Notation $\lceil\cdot\rceil$ is defined as the absolute value of a matrix (vector); specifically, for a matrix (vector) $V$, its absolute value matrix (vector) is

$$
\lceil V\rceil=\left[\begin{array}{cccc}
\left|v_{11}\right| & \left|v_{12}\right| & \cdots & \left|v_{1 n}\right| \\
\left|v_{21}\right| & \left|v_{22}\right| & \cdots & \left|v_{2 n}\right| \\
\vdots & \vdots & \ddots & \vdots \\
\left|v_{m 1}\right| & \left|v_{m 2}\right| & \cdots & \left|v_{m n}\right|
\end{array}\right]
$$

Lemma 1 For two conformable matrices (vectors) $V_{1}$ and $V_{2}$, if $V_{1}\left(V_{2}\right)$ is diagonal, then $\left\lceil V_{1} V_{2}\right\rceil=\left\lceil V_{1}\right\rceil\left\lceil V_{2}\right\rceil$; furthermore, if all the elements of diagonal matrix $V_{1}\left(V_{2}\right)$ are positive, it has $\left\lceil V_{1} V_{2}\right\rceil=V_{1}\left\lceil V_{2}\right\rceil\left(\left\lceil V_{1} V_{2}\right\rceil=\left\lceil V_{1}\right\rceil V_{2}\right)$. The proof is only too evident.

Theorem 2 For the reaching law (17), if $q_{1 i}$ are selected to satisfy $q_{1 i} h \ll 2$ and adaptive switching gains $q_{2 i}(k), i=$ $1, \ldots, m$, are designed as

$$
\begin{aligned}
& {\left[q_{21}(k), q_{22}(k), \ldots, q_{2 m}(k)\right]^{\mathrm{T}}} \\
& =K_{2}+C_{2}^{-1}\left\lceil C_{1} L^{-1}+G\right\rceil\left\lceil\hat{d}_{u}(k)-\hat{d}_{u}(k-1)\right\rceil,
\end{aligned}
$$

then $s(k)$ will eventually converge to a QSM band as

$\Omega \triangleq\left\{s_{i}(k)|| s_{i}(k) \mid \leq \mu_{i}, i=1, \ldots, m\right\}$,

where $\mu_{i} \triangleq 2 q_{2 i}(k) h=O\left(h^{2}\right)$, and the attractiveness region of sliding surface (12) can be constructed as

$\Omega_{1} \triangleq\left\{s_{i}(k)|| s_{i}(k) \mid>\frac{2 q_{2 i}(k) h}{2-q_{1 i} h}, i=1, \ldots, m\right\}$.

Proof The designed adaptive switching gain (19) will be first testified to fulfill the inequality (18). In view of (9), it is demonstrated that

$$
\begin{aligned}
& \left|\left(C_{1}+G L\right)\left(d_{u}(k)-\hat{d}_{u}(k)\right)+C_{2} d_{m}(k)\right|_{i} \\
& \leq\left\{\left\lceil C_{1}+G L\right\rceil\left\lceil d_{u}(k)-\hat{d}_{u}(k)\right\rceil+\left\lceil C_{2} d_{m}(k)\right\rceil\right\}_{i} \\
& =\left\{\left\lceil C_{1}+G L\right\rceil\left\lceil\left(I_{n-m}-L\right)^{k} d_{u}(0)\right\rceil+\left\lceil C_{2} d_{m}(k)\right\rceil\right\}_{i} \\
& \leq\left\{\left\lceil C_{1}+G L\right\rceil\left\lceil\left(I_{n-m}-L\right)^{k-1} d_{u}(0)\right\rceil+\left\lceil C_{2} K_{2}\right\rceil\right\}_{i} \\
& =\left\{\left\lceil C_{1}+G L\right\rceil\left\lceil d_{u}(k-1)-\hat{d}_{u}(k-1)\right\rceil+h K_{2}\right\}_{i} \\
& =\left\{\left\lceil C_{1}+G L\right\rceil L^{-1}\left\lceil\hat{d}_{u}(k)-\hat{d}_{u}(k-1)\right\rceil+h K_{2}\right\}_{i} \\
& =\left\{\left\lceil C_{1} L^{-1}+G\right\rceil\left\lceil\hat{d}_{u}(k)-\hat{d}_{u}(k-1)\right\rceil+h K_{2}\right\}_{i},
\end{aligned}
$$

where \{\}$_{i}$ is the $i$ th value of a column vector. The adaptive gain terms $q_{2 i}(k)$ can counteract the effect of matched and unmatched disturbances on discrete reaching law (17), and $Q_{2}(k)$ can automatically adjust its value according to the output of RODO. As the estimation error of (7) converges into $\Psi$, i.e., $\left|d_{u}(k-1)-\hat{d}_{u}(k-1)\right|_{i} \leq \varepsilon_{i} / \lambda_{i}$, then $q_{2 i}(k) \leq$ $\left|C_{2}^{-1}\left(C_{1} L^{-1}+G\right) \varepsilon+K_{2}\right|_{i}$. Since $C_{2}=O(h), \varepsilon=O\left(h^{2}\right)$, and $K_{2}=O(h)$, it is deduced that $q_{2 i}(k)=O(h)$ in steady state. For reaching law (17), choosing Lyapunov function $V_{i}(k)=$ $s_{i}^{2}(k)$ and defining $\Delta V_{i}(k)=V_{i}(k+1)-V_{i}(k), \Delta V_{i}(k)<0$ is equivalent to

$$
\begin{aligned}
& \{(s(k+1)-s(k)) \operatorname{sgn}(s(k))\}_{i} \\
= & \left\{-Q_{1} h|s(k)|-Q_{2}(k) h+C_{2} d_{m}(k) \operatorname{sgn}(s(k))\right. \\
& \left.+\left(C_{1}+G L\right)\left(d_{u}(k)-\hat{d}_{u}(k)\right) \operatorname{sgn}(s(k))\right\}_{i} \\
= & \left\{-Q_{1} h|s(k)|-\left\lceil C_{1} L^{-1}+G\right\rceil\left\lceil\hat{d}_{u}(k)-\hat{d}_{u}(k-1)\right\rceil\right. \\
& +\left(C_{1}+G L\right)\left(d_{u}(k)-\hat{d}_{u}(k)\right) \operatorname{sgn}(s(k)) \\
& \left.-h K_{2}+h d_{m}(k) \operatorname{sgn}(s(k))\right\}_{i}<0,
\end{aligned}
$$

and

$$
\begin{aligned}
& \{(s(k+1)+s(k)) \operatorname{sgn}(s(k))\}_{i} \\
= & \left\{\left(2 I_{m}-Q_{1} h\right)|s(k)|-Q_{2}(k) h+C_{2} d_{m}(k) \operatorname{sgn}(s(k))\right. \\
& \left.+\left(C_{1}+G L\right)\left(d_{u}(k)-\hat{d}_{u}(k)\right) \operatorname{sgn}(s(k))\right\}_{i} \\
= & \left\{\left(2 I_{m}-Q_{1} h\right)|s(k)|-2 Q_{2}(k) h+h d_{m}(k) \operatorname{sgn}(s(k))\right. \\
& +h K_{2}+\left\lceil C_{1} L^{-1}+G\right\rceil\left\lceil\hat{d}_{u}(k)-\hat{d}_{u}(k-1)\right\rceil \\
& \left.+\left(C_{1}+G L\right)\left(d_{u}(k)-\hat{d}_{u}(k)\right) \operatorname{sgn}(s(k))\right\}_{i}>0 .
\end{aligned}
$$

When the system trajectory does not cross the sliding surface, equation (23) can ensure that it moves towards $s(k)=$ 0 ; equation (24) makes the system trajectory attenuated if it moves across the sliding surface. It is easy to verify that (23) is satisfied globally, while only in region $\left|s_{i}(k)\right|>2 q_{2 i}(k) h /$ $\left(2-q_{1 i} h\right)=O\left(h^{2}\right)$, it has

$\{(s(k+1)+s(k)) \operatorname{sgn}(s(k))\}_{i}$

$$
\geq\left\{\left(2 I_{m}-Q_{1} h\right)|s(k)|-2 Q_{2}(k) h\right\}_{i}>0 \text {, }
$$

i.e., the sliding variable $s(k)$ meets the reaching condition in the attractiveness region $\Omega_{1}$. For system states outside $\Omega_{1}$, two cases are discussed.

Case 1: For the case of $0<s_{i}(k) \leq 2 q_{2 i}(k) h /\left(2-q_{1 i} h\right)$, according to the reaching law (17), it gives

$$
-2 q_{2 i}(k) h<s_{i}(k+1) \leq \frac{2 q_{2 i}(k)\left(1-q_{1 i} h\right) h}{2-q_{1 i} h} .
$$

Case 2: If $-2 q_{2 i}(k) h /\left(2-q_{1 i} h\right) \leq s_{i}(k)<0$, similarly, it can be derived that

$$
-\frac{2 q_{2 i}(k)\left(1-q_{1 i} h\right) h}{2-q_{1 i} h} \leq s_{i}(k+1)<2 q_{2 i}(k) h .
$$

Since $2 q_{2 i}(k)\left(1-q_{1 i} h\right) h /\left(2-q_{1 i} h\right)<2 q_{2 i}(k) h$, we can infer that the system states outside $\Omega_{1}$ can reach $\left|s_{i}(k)\right|=2 q_{2 i}(k) h$ at most. The system states lying in region $2 q_{2 i}(k) h /(2-$ $\left.q_{1 i} h\right)<\left|s_{i}(k)\right| \leq 2 q_{2 i} h$ satisfy the reaching condition, and continue to move towards sliding surface, i.e., once $s(k)$ enters the region $\Omega$, it cannot escape from the region. Therefore, the QSM band can be obtained as (20); furthermore, $\mu_{i}=2 q_{2 i}(k) h=O\left(h^{2}\right)$. 
Remark 3 The general reaching law-based DSMC with disturbance compensation for unmatched systems has been reported in [7]. However, owing to using constant switching gain, the boundary layer of the QSM band is only in the order of $O(h)$. In this paper, it can be seen from (19) that not only the current disturbance estimation $\hat{d}(k)$ but also the previous disturbance information $\hat{d}(k-1)$ is utilized to design the adaptive switching gain to significantly reduce the width of QSM band. Based on the proposed RODO, the adaptive reaching law (17) can realize the boundary layer $s(k)=O\left(h^{2}\right)$ for the final sliding mode motion, even though there exist unmatched disturbances in the discrete-time system (1), which means that the chattering effect is attenuated.

\subsection{Control law design}

Based on the RODO and adaptive reaching law mentioned above, the discrete sliding mode controller can be described in the following theorem.

Theorem 3 For the discrete-time system (1) with sliding surface (12), by applying the reaching law (17), the discrete sliding mode controller can be designed as

$$
\begin{aligned}
u(k)= & C_{2}^{-1}\left[\left(I_{m}-Q_{1} h\right) s(k)-Q_{2}(k) h \operatorname{sgn}(s(k))\right. \\
& -\left(C_{1} \Phi_{11}+C_{2} \Phi_{21}\right) x_{1}(k)-\left(C_{1} \Phi_{12}+C_{2} \Phi_{22}\right) x_{2}(k) \\
& \left.-\left(C_{1}+G\right) \hat{d}_{u}(k)\right] .
\end{aligned}
$$

Proof In consideration of (3) and (12), it is derived that

$$
\begin{aligned}
s(k+1)=C_{1} x_{1}(k+1)+C_{2} x_{2}(k+1)+G \hat{d}_{u}(k+1) \\
=C_{1}\left(\Phi_{11} x_{1}(k)+\Phi_{12} x_{2}(k)+d_{u}(k)\right)+G \hat{d}_{u}(k+1) \\
\quad+C_{2}\left(\Phi_{21} x_{1}(k)+\Phi_{22} x_{2}(k)+u(k)+d_{m}(k)\right) \\
=C_{1}\left(\Phi_{11} x_{1}(k)+\Phi_{12} x_{2}(k)\right)+C_{2}\left(\Phi_{21} x_{1}(k)+\Phi_{22} x_{2}(k)\right) \\
\quad+C_{1} d_{u}(k)+C_{2} u(k)+C_{2} d_{m}(k)+G \hat{d}_{u}(k+1) .
\end{aligned}
$$

Combining reaching law (17) and (29), the expression of controller can be further deduced as:

$$
\begin{aligned}
u(k)= & C_{2}^{-1}\left[\left(I_{m}-Q_{1} h\right) s(k)-Q_{2}(k) h \operatorname{sgn}(s(k))\right. \\
& -\left(C_{1} \Phi_{11}+C_{2} \Phi_{21}\right) x_{1}(k)-\left(C_{1} \Phi_{12}+C_{2} \Phi_{22}\right) x_{2}(k) \\
& \left.-\left(C_{1}+G L\right) \hat{d}_{u}(k)-G\left(\hat{d}_{u}(k+1)-L d_{u}(k)\right)\right] .
\end{aligned}
$$

From (9), it can be obtained that $\hat{d}_{u}(k+1)-L d_{u}(k)=\left(I_{n-m}-\right.$ $L) \hat{d}_{u}(k)$; then by substituting it into (30), the control law (28) can be generated.

It is observed from (28) that the magnitude of $u(k)$ may be large as a result of initial estimation error of RODO. However, an over-large control input may be undesirable in the case of actuator saturation constraint. To avoid large chattering at the transient process, the implementation of
(28) in real practice can be replace by a saturation function as follows:

$U(k)= \begin{cases}\kappa \cdot \operatorname{sgn}(u(k)), & |u(k)|>\kappa, \\ u(k), & |u(k)| \leq \kappa,\end{cases}$

where $\kappa=\left[\kappa_{1}, \kappa_{2}, \ldots, \kappa_{m}\right]^{\mathrm{T}}$ represent saturation values that input actuators can reach.

Remark 4 It should be pointed out that in general, the reaching law method cannot be used for discrete sliding surfaces that contain disturbance compensation; otherwise, $\hat{d}_{u}(k+1)$ will be included in the expression of $u(k)$, which is impossible to realize in the actual system. This problem is solved by the proposed RODO. The disturbance estimation provided by the RODO is utilized in the sliding surface, reaching law, and adaptive switching gain. Accordingly, this paper makes full use of the RODO such that it has the function of disturbance prediction to make it possible to run adaptive reaching law algorithm here.

Remark 5 The whole control strategy enjoys the advantages of low computational complexity and less conservativeness. For the sliding surface (12), only $(n-m)$-dimension $C_{1}$ requires to be calculated by using pole placement. The RODO can accurately estimate the unmatched disturbance in real time, and thus the priori boundedness of unmatched disturbance does not need to be known. For the reaching law (17), the switching gain adaptively adjusts its value to ensure sliding mode motion and to avoid overestimation of disturbance simultaneously. When the closed-loop system is in steady state, it has $q_{2 i}(k) \leq\left|C_{2}^{-1}\left(C_{1} L^{-1}+G\right) \varepsilon+K_{2}\right|_{i}=O(h)$, and $\left|s_{i}(k)\right| \leq 2 q_{2 i}(k) h=O\left(h^{2}\right)$, in which the minimum value of switching gain can be obtained. Therefore, the proposed control scheme is less conservative.

\section{Simulation results}

The bank-to-turn (BTT) missile has become more and more popular due to its long-range maneuverability and high precision. To demonstrate the effectiveness of the proposed control strategy in this paper, the pitch/yaw dynamic model of a BTT missile is considered in this section $[8,24]$. Taking roll rate as a parameter of the pitch/yaw model, the differential equations are given as

$$
\left\{\begin{aligned}
\dot{\alpha}= & \omega_{z}-\frac{1}{57.3} \omega_{x} \beta-e_{4} \alpha-e_{5} \delta_{z}+d_{\alpha}, \\
\dot{\beta}= & \omega_{y}+\frac{1}{57.3} \omega_{x} \alpha-f_{4} \beta-f_{5} \delta_{y}+d_{\beta}, \\
\dot{\omega}_{z}= & -\left(e_{1}+g_{1}\right) \omega_{z}+\left(g_{1} e_{4}-e_{2}\right) \alpha+\frac{g_{1}}{57.3} \omega_{x} \beta \\
& +\left(-g_{1} e_{5}-e_{3}\right) \delta_{z}+\frac{J_{x}-J_{y}}{57.3 J_{z}} \omega_{x} \omega_{y}+d_{\omega_{z}}, \\
\dot{\omega}_{y}= & -\left(f_{1}+g_{2}\right) \omega_{y}+\left(g_{2} f_{4}-f_{2}\right) \beta-\frac{g_{2}}{57.3} \omega_{x} \alpha \\
& +\left(g_{2} f_{5}-f_{3}\right) \delta_{y}+\frac{J_{z}-J_{x}}{57.3 J_{y}} \omega_{x} \omega_{z}+d_{\omega_{y}},
\end{aligned}\right.
$$


Table 1 Time-varying parameters of a BTT missile

\begin{tabular}{lcccr}
\hline Parameters & $0-4.5 \mathrm{~s}$ & $4.5 \mathrm{~s}-11.7 \mathrm{~s}$ & $11.7 \mathrm{~s}-19.5 \mathrm{~s}$ & $19.5 \mathrm{~s}-25 \mathrm{~s}$ \\
\hline$e_{1}$ & 1.59 & 1.49 & 1.27 & 1.13 \\
$e_{2}$ & 260.56 & 266.42 & 196.74 & 137.39 \\
$e_{3}$ & 185.49 & 182.53 & 176.93 & 160.89 \\
$e_{4}$ & 1.51 & 1.29 & 1.17 & 1.13 \\
$e_{5}$ & 0.30 & 0.24 & 0.22 & 0.19 \\
$f_{1}$ & 1.66 & 1.50 & 1.27 & 1.13 \\
$f_{2}$ & 39.99 & -24.63 & -31.45 & -41.43 \\
$f_{3}$ & 159.97 & 170.53 & 182.03 & 184.09 \\
$f_{4}$ & 0.77 & 0.65 & 0.68 & 0.69 \\
$f_{5}$ & 0.25 & 0.19 & 0.19 & 0.18 \\
$g_{1}$ & 0.29 & 0.19 & 0.15 & 0.12 \\
$g_{2}$ & 0.30 & 0.20 & 0.15 & 0.12 \\
\hline
\end{tabular}

where $\alpha$ and $\beta$ are the angle-of-attack and the sideslip angle; $\omega_{x}, \omega_{y}$ and $\omega_{z}$ represent roll, yaw, and pitch rates respectively; $\delta_{y}$ and $\delta_{z}$ denote yaw and pitch control deflection angles; $d_{\alpha}, d_{\beta}, d_{\omega_{z}}, d_{\omega_{y}}$ are disturbances on four differential equations; $J_{x}, J_{y}$ and $J_{z}$ are roll, yaw, and pitch moments of inertia; $e_{i}, f_{i}(i=1, \ldots, 5), g_{1}$, and $g_{2}$ are time-varying parameters of BTT systems, which are listed in Table 1.The state space model is established as

$$
\begin{aligned}
\dot{x}(t)= & {\left[\begin{array}{cccc}
-e_{4} & \frac{-\omega_{x}}{57.3} & 1 & 0 \\
\frac{\omega_{x}}{57.3} & -f_{4} & 0 & 1 \\
g_{1} e_{4}-e_{2} & \frac{g_{1} \omega_{x}}{57.3} & -e_{1}-g_{1} & \frac{\left(J_{x}-J_{y}\right) \omega_{x}}{57.3 J_{z}} \\
\frac{-g_{2} \omega_{x}}{57.3} & g_{2} f_{4}-f_{2} & \frac{\left(J_{z}-J_{x}\right) \omega_{x}}{57.3 J_{y}} & -f_{1}-g_{2}
\end{array}\right] x(t) } \\
& +\left[\begin{array}{cc}
-e_{5} & 0 \\
0 & -f_{5} \\
-g_{1} e_{5}-e_{3} & 0 \\
0 & g_{2} f_{5}-f_{3}
\end{array}\right] u(t)+d(t)
\end{aligned}
$$

where the state vector $x(t)=\left[\alpha, \beta, \omega_{z}, \omega_{y}\right]^{\mathrm{T}}$, the output $y(t)=$ $[\alpha, \beta]^{\mathrm{T}}$, the control input $u(t)=\left[\delta_{z}, \delta_{y}\right]^{\mathrm{T}}$, and the disturbance vector $d(t)=\left[d_{\alpha}, d_{\beta}, d_{\omega_{z}}, d_{\omega_{y}}\right]^{\mathrm{T}}$. Some parameters of BTT missiles are taken from [24] as follows: $J_{x}=100 \mathrm{~kg} . \mathrm{m}^{2}$, $J_{y}=5700 \mathrm{~kg} \cdot \mathrm{m}^{2}$ and $J_{z}=5600 \mathrm{~kg} \cdot \mathrm{m}^{2}$. The roll rate is supposed as $\omega_{x}=400^{\circ} / \mathrm{s}$. Choosing the sampling time $h=1 \mathrm{~ms}$, the discrete state matrices, corresponding to four time segments $0-4.5 \mathrm{~s}, 4.5 \mathrm{~s}-11.7 \mathrm{~s}, 11.7 \mathrm{~s}-19.5 \mathrm{~s}$ and $19.5 \mathrm{~s}-25 \mathrm{~s}$, can be got as

$$
\begin{aligned}
& \Phi_{11}^{1}=\left[\begin{array}{rr}
0.999 & -0.007 \\
0.007 & 0.999
\end{array}\right], \Phi_{12}^{1}=10^{-4}\left[\begin{array}{rr}
-1.854 & 165.6 \\
-201.5 & -1.599
\end{array}\right], \\
& \Phi_{21}^{1}=\left[\begin{array}{rr}
1.401 & -0.0156 \\
0.014 & 0.249
\end{array}\right], \Phi_{22}^{1}=\left[\begin{array}{rr}
0.997 & -0.006 \\
0.008 & 0.998
\end{array}\right], \\
& \Phi_{11}^{2}=\left[\begin{array}{rr}
0.999 & -0.007 \\
0.007 & 0.999
\end{array}\right], \Phi_{12}^{2}=10^{-4}\left[\begin{array}{rr}
-1.824 & 153.4 \\
-220.6 & -1.704
\end{array}\right], \\
& \Phi_{21}^{2}=\left[\begin{array}{rr}
1.457 & -0.0124 \\
0.0075 & -0.145
\end{array}\right], \Phi_{22}^{2}=\left[\begin{array}{rr}
0.998 & -0.0065 \\
0.007 & 0.998
\end{array}\right],
\end{aligned}
$$
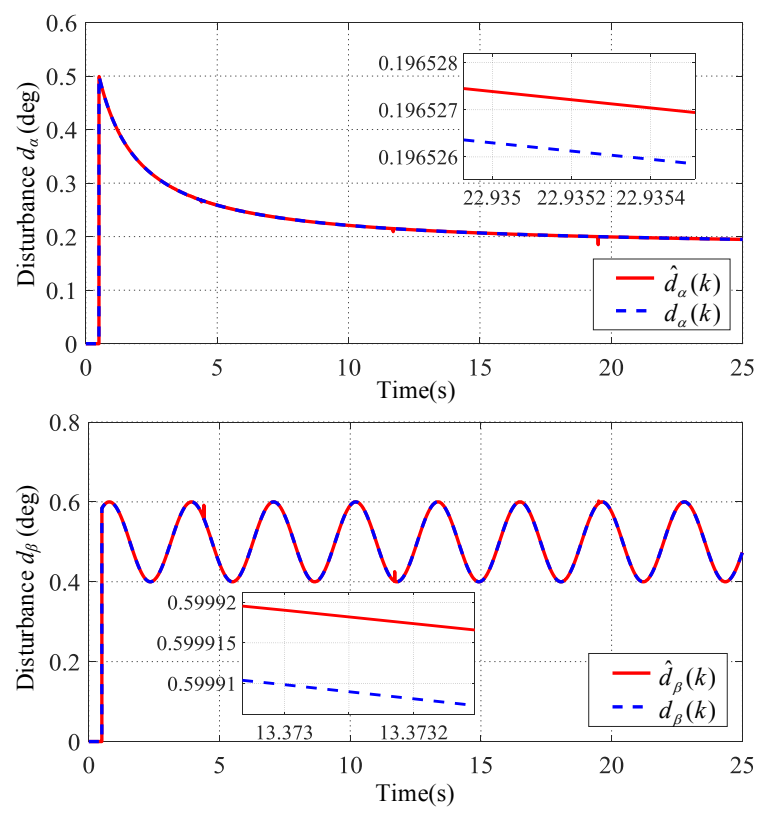

Fig. 1 Simulation results on disturbance estimation of the RODO.

$$
\begin{aligned}
& \Phi_{11}^{3}=\left[\begin{array}{rr}
0.999 & -0.007 \\
0.007 & 0.999
\end{array}\right], \Phi_{12}^{3}=10^{-4}\left[\begin{array}{rr}
-1.768 & 165.9 \\
-211.3 & -1.819
\end{array}\right], \\
& \Phi_{21}^{3}=\left[\begin{array}{rr}
1.110 & -0.0097 \\
0.005 & -0.173
\end{array}\right], \Phi_{22}^{3}=\left[\begin{array}{rr}
0.998 & -0.0072 \\
0.0065 & 0.998
\end{array}\right], \\
& \Phi_{11}^{4}=\left[\begin{array}{rr}
0.999 & -0.007 \\
0.007 & 0.999
\end{array}\right], \Phi_{12}^{4}=10^{-4}\left[\begin{array}{rr}
-1.607 & 167.1 \\
-192.5 & -1.840
\end{array}\right], \\
& \Phi_{21}^{4}=\left[\begin{array}{rr}
0.853 & -0.008 \\
0.0037 & -0.225
\end{array}\right], \Phi_{22}^{4}=\left[\begin{array}{rr}
0.998 & -0.008 \\
0.006 & 0.999
\end{array}\right] .
\end{aligned}
$$

The zero drifts are inevitable during the measurement of yaw and pitch rates; assume that angle-of-attack and sideslip angle channels have exponential and sinusoidal disturbances respectively; then they are set as

$$
\begin{aligned}
& d_{\alpha}(k)= \begin{cases}0, & 0 \leq k h<0.5 \mathrm{~s}, \\
0.5\left(1-e^{\frac{1}{2}}+e^{\frac{1}{1.5+k h}}\right) & 0.5 \mathrm{~s} \leq k h<25 \mathrm{~s},\end{cases} \\
& d_{\beta}(k)= \begin{cases}0, & 0 \leq k h<0.5 \mathrm{~s}, \\
0.5+0.1 \sin (\pi k h), & 0.5 \mathrm{~s} \leq k h<25 \mathrm{~s},\end{cases} \\
& d_{\omega_{z}}(k)=0.02^{\circ} / \mathrm{s}, d_{\omega_{y}}(k)=0.02^{\circ} / \mathrm{s} .
\end{aligned}
$$

It follows from (11) that the estimation error of RODO converges to $\Psi$ with exponential rates $\left|1-\lambda_{1}\right|^{k} d_{\alpha}(0)$ and $\mid 1-$ $\left.\lambda_{2}\right|^{k} d_{\beta}(0)$. Obviously, the smaller the values of $\left|1-\lambda_{1}\right|$ and $\left|1-\lambda_{2}\right|$ are, the faster the convergence speed of RODO is; accordingly, the values of $\lambda_{1}$ and $\lambda_{2}$ should be designed to be close to 1 . Here the gain matrix $L$ is selected as $\operatorname{diag}\{0.8,0.9\}$. From (15) and (16), the parameter matrices $C_{1}$ and $G$ of the sliding surface corresponding to four time segments are designed as

$$
C_{1}^{1}=\left[\begin{array}{r}
-5.403-0.096 \\
0.146-5.603
\end{array}\right], G^{1}=\left[\begin{array}{rr}
-5.394 & -0.056 \\
0.068 & -6.252
\end{array}\right],
$$



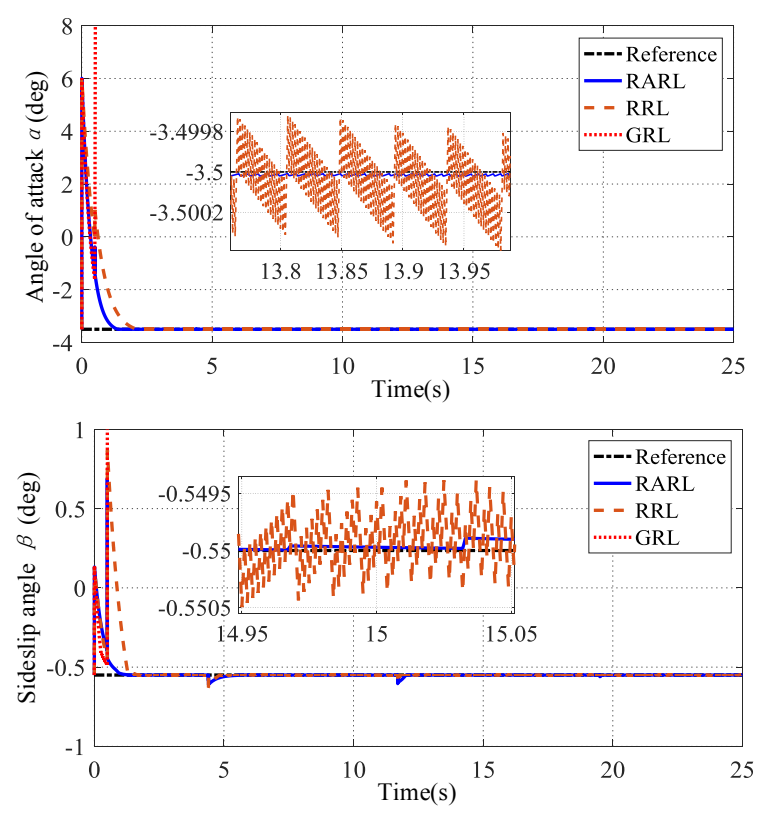

Fig. 2 Output responses for the BTT systems with unmatched disturbances by applying controllers (31), (32) and (33).
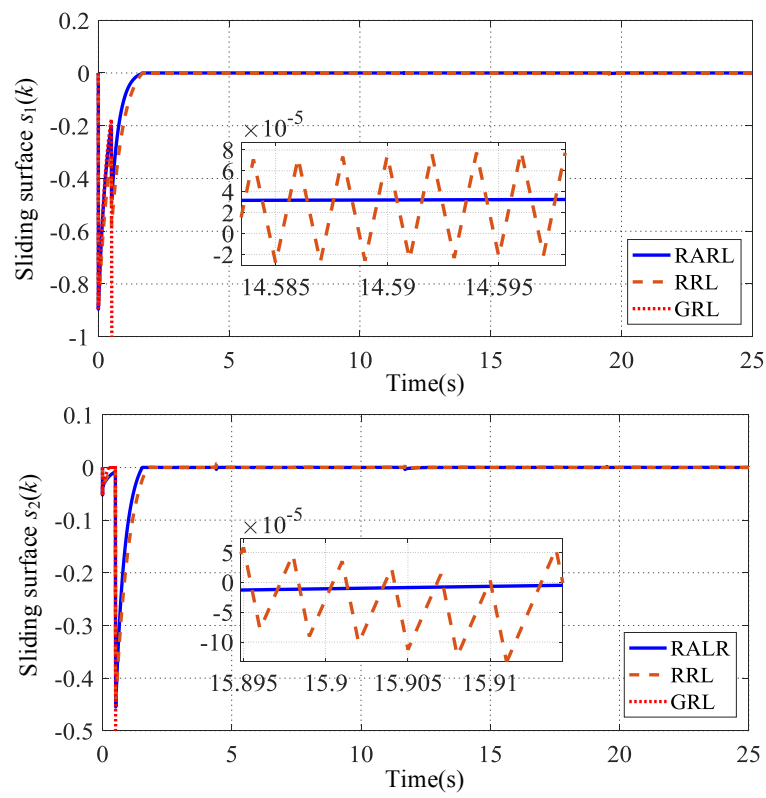

Fig. 3 Sliding surfaces for the BTT systems with unmatched disturbances by applying controllers (31), (32) and (33).

$$
\begin{aligned}
& C_{1}^{2}=\left[\begin{array}{rr}
-5.479 & -0.019 \\
0.119 & -5.275
\end{array}\right], G^{2}=\left[\begin{array}{r}
-5.482-0.049 \\
0.071-5.867
\end{array}\right], \\
& C_{1}^{3}=\left[\begin{array}{rr}
-5.106 & 0.087 \\
0.131 & -5.476
\end{array}\right], G^{3}=\left[\begin{array}{r}
-5.657-0.052 \\
0.065-5.498
\end{array}\right], \\
& C_{1}^{4}=\left[\begin{array}{r}
-5.578-0.083 \\
0.145-5.446
\end{array}\right], G^{4}=\left[\begin{array}{r}
-6.223-0.057 \\
0.065-5.437
\end{array}\right] .
\end{aligned}
$$

In order to show the effectiveness of the proposed RODObased adaptive reaching law (RARL) control for BTT systems with unmatched disturbances, the general reaching law
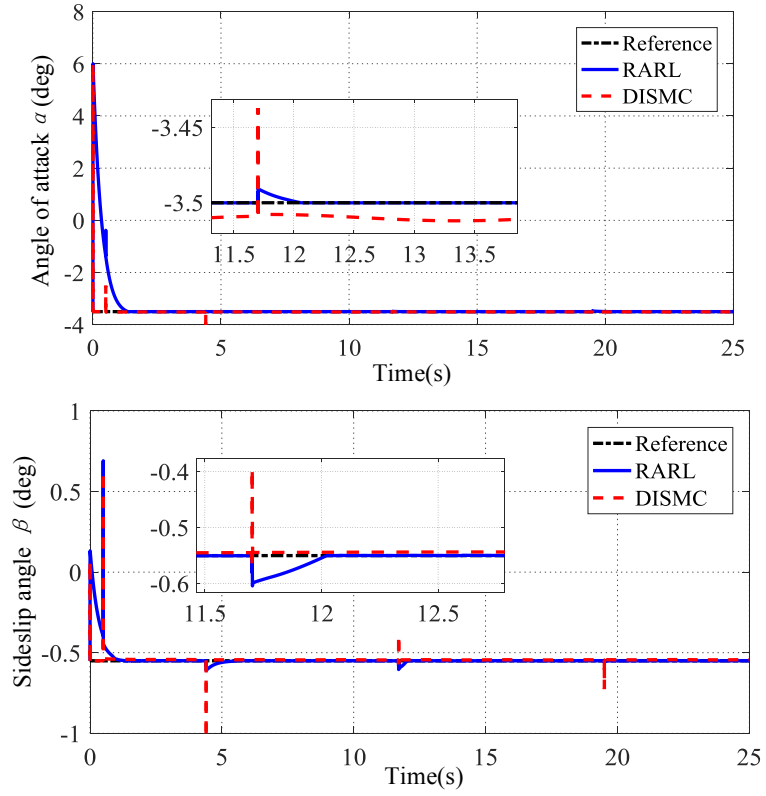

Fig. 4 Output responses for the BTT systems with unmatched disturbances by applying controllers (31) and (35).
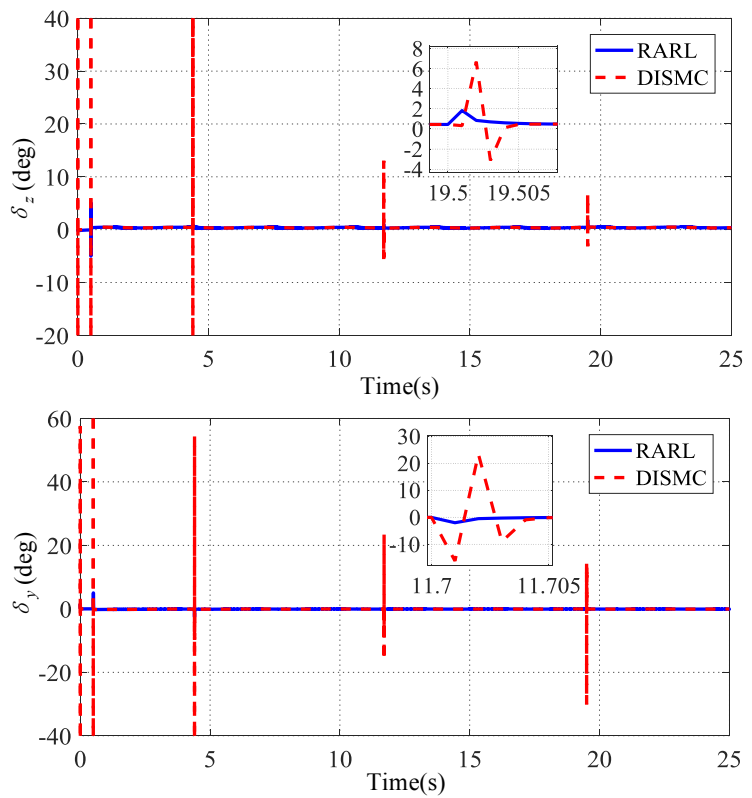

Fig. 5 Input controls for the BTT systems with unmatched disturbances by applying controllers (31) and (35).

(GRL) and RODO-based reaching law (RRL) are also adopted, and their corresponding controllers are given as

$$
\begin{aligned}
u_{1}(k)= & C_{2}^{-1}\left[\left(I_{2}-Q_{1} h\right) s(k)-Q_{3} h \operatorname{sgn}(s(k))\right. \\
& \left.-\left(C_{1} \Phi_{11}+C_{2} \Phi_{21}\right) x_{1}(k)-\left(C_{1} \Phi_{12}+C_{2} \Phi_{22}\right) x_{2}(k)\right],
\end{aligned}
$$

$$
\begin{aligned}
u_{2}(k)= & C_{2}^{-1}\left[\left(I_{2}-Q_{1} h\right) s(k)-Q_{3} h \operatorname{sgn}(s(k))-\left(C_{1}+G\right) \hat{d}_{u}(k)\right. \\
& \left.-\left(C_{1} \Phi_{11}+C_{2} \Phi_{21}\right) x_{1}(k)-\left(C_{1} \Phi_{12}+C_{2} \Phi_{22}\right) x_{2}(k)\right] .
\end{aligned}
$$


Table 2 Steady-state errors of the four control methods

\begin{tabular}{lcccr}
\hline Steady-state error $\left({ }^{\circ}\right)$ & GRL & RRL & RARL & DISMC \\
\hline$|\Delta \alpha(\infty)|$ & 62 & 0.0018 & $1.0 \times 10^{-5}$ & 0.012 \\
$|\Delta \beta(\infty)|$ & 195 & 0.0012 & $2.1 \times 10^{-4}$ & 0.0071 \\
\hline
\end{tabular}

The parameters of controllers (31), (32) and (33) are selected as $\kappa=\left[3^{\circ}, 3^{\circ}\right]^{\mathrm{T}}, Q_{1}=\operatorname{diag}\{3,3\}$ and $Q_{3}=\operatorname{diag}\{0.1,0.1\}$. The initial condition is set as $x(0)=\left[-6^{\circ}, 0,0,0\right]^{\mathrm{T}}$, and the expectations of angle-of-attack and sideslip angle are $\alpha^{*}=$ $-3.5^{\circ}$ and $\beta^{*}=-0.55^{\circ}$. The simulation results are presented in Figs. 1, 2 and 3.

As shown in Fig. 1, the RODO can quickly track real disturbances, and the estimation errors are in the order of $10^{-6}$ for exponential disturbance, $10^{-5}$ for sinusoidal disturbance, i.e., the unmatched disturbances can be estimated by RODO very well. It can be seen from Figs. 2 and 3 that the proposed RARL-based controller exhibits much better dynamic and static performances than the other two methods. Specifically, the system states and sliding variables under the GRL-based controller are divergent; the RRL-based control approach can guarantee the convergence of the BTT system, but severe chattering also exists; the RARL method can achieve smaller QSM band such that chattering effect is significantly reduced.

Moreover, for the purpose of comparison, the DISMC method is employed [1,27], and the corresponding discrete integral sliding surface is chosen as

$\left\{\begin{array}{l}\delta(k)=D x(k)-D x(0)+\sigma(k), \\ \sigma(k)=\sigma(k-1)+E x(k-1),\end{array}\right.$

where $\delta(k), \sigma(k) \in R^{m}, D, E \in R^{m \times n}$. The parameter matrices are designed as $R=\left[\left(\Phi-I_{n}\right) Q\left(\Phi-I_{n}\right)^{\mathrm{T}}\right]^{-1}, P=I_{n}-$ $\Gamma\left(\Gamma^{\mathrm{T}} R \Gamma\right)^{-1} \Gamma^{\mathrm{T}} R, E=-D\left(\Phi-I_{n}-\Gamma F\right), D=\left[0_{m \times n}, I_{m}\right]$. $\left[\left(\Phi-I_{n}-\Gamma F\right)\left(\Phi-I_{n}\right)^{-1} P, \Gamma\right]^{+}$, where $[\cdot]^{+}$is left pseudoinverse of a matrix, $Q \in R^{n}$ is weight matrix of system states, and $F \in R^{m \times n}$ should be designed to make all the poles of $(\Phi-\Gamma F)$ within the unit circle. Then the DISMC law is further given as

$u_{I}(k)=-(D \Gamma)^{-1}[(D \Phi-D+E) x(k)+\delta(k)]$.

Selecting $Q=\operatorname{diag}\left\{10,10,10^{-5}, 10^{-5}\right\}$, the simulation conditions are the same as above, and then the simulation results of BTT missile systems controlled by (31) and (35) are shown in Figs. 4 and 5.

It can be observed from Fig. 4 that the RARL-base control method can precisely track the given expectation during the whole process of missile flight regardless of unmatched disturbances, while DISMC approach drives the system states to the desired expectation with large steady-state error, and the former has smaller overshoot when the parameters of BTT missiles change. As shown in Fig. 5, the magnitude of input control of RARL is much smaller than DISMC law, i.e., the proposed method produces attenuated chattering. In addition, for DISMC, more parameter matrices need to be determined, which leads to the increase of computational load, especially for higher dimensional system; pole assignment technique is used to design $n$-dimensional $F$ for DISMC law, but only $(n-m)$-dimensional $C_{1}$ for proposed RARL, i.e., the proposed control algorithm in this paper is much simpler than DISMC. Finally, the steady-state errors $|\Delta \alpha(\infty)|$ of angle-of-attack and $|\Delta \beta(\infty)|$ of the sideslip angle for the four control schemes are presented in Table 2. From the simulation results, we can see that the proposed RARL-based control algorithm is verified to have higher control precision and better unmatched disturbance rejection ability than the traditional methods.

\section{Conclusion}

For discretized multi-input multi-output systems under unmatched disturbances, this paper has developed a reducedorder disturbance observer based adaptive discrete sliding mode control approach. The RODO can fast converge to real unknown unmatched disturbance, and the designed sliding surface containing the disturbance estimation can drive system states to the desired equilibrium point. The adaptive reaching law has been established to reduce the quasi sliding mode band. It should be noted that the matched disturbance has to satisfy the bounded constraint for proposed control algorithm. In future research, the more general control law for discrete-time systems with unmatched uncertainties will be paid attention to.

\section{Declarations}

The authors have no conflicts of interest to declare that are relevant to the content of this article. All data generated or analysed during the current study are available from the corresponding author on reasonable request.

\section{References}

1. Abidi, K., Xu, J.X., Yu, X.: On the discrete-time integral slidingmode control. IEEE Trans. Autom. Control 52(4), 709-715 (2007)

2. Behera, A.K., Bandyopadhyay, B.: Steady-state behaviour of discretized terminal sliding mode. Automatica 54, 176-181 (2015)

3. Cao, W.J., Xu, J.X.: Nonlinear integral-type sliding surface for both matched and unmatched uncertain systems. IEEE Trans. Autom. Control 49(8), 1355-1360 (2004)

4. Chen, W.H.: Nonlinear disturbance observer-enhanced dynamic inversion control of missiles. J. Guid. Control Dyn 26(1), 161166 (2003)

5. Chen, W.H., Ballance, D.J., Gawthrop, P.J., O’Reilly, J.: A nonlinear disturbance observer for robotic manipulators. IEEE Trans. Ind. Electron 47(4), 932-938 (2000) 
6. Choi, H.H.: LMI-based sliding surface design for integral sliding mode control of mismatched uncertain systems. IEEE Trans. Autom. Control 52(4), 736-742 (2007)

7. Du, H., Wen, G., Cheng, Y., Lu, W., Huang, T.: Designing discretetime sliding mode controller with mismatched disturbances compensation. IEEE Trans. Ind. Inform 16(6), 4109-4118 (2019)

8. Duan, G., Wang, H., Zhang, H.: Parameter design of smooth switching controller and application for bank-to-turn missiles. Aerospace Control 23(2), 41-46 (2005)

9. Feng, Y., Zhou, M., Zheng, X., Han, F., Yu, X.: Full-order terminal sliding-mode control of MIMO systems with unmatched uncertainties. J. Frankl. Inst 355(2), 653-674 (2018)

10. Fridman, L., Moreno, J., Iriarte, R.: Sliding Modes after the First Decade of the 21st Century. Springer-Verlag, Berlin (2011)

11. Gao, W., Wang, Y., Homaifa, A.: Discrete-time variable structure control systems. IEEE Trans. Ind. Electron 42(2), 117-122 (1995)

12. Gholami, A., Markazi, A.H.: A new adaptive fuzzy sliding mode observer for a class of MIMO nonlinear systems. Nonlinear Dyn 70(3), 2095-2105 (2012)

13. Han, H., Wu, X., Qiao, J.: Design of robust sliding mode control with adaptive reaching law. IEEE Trans. Syst Man. Cybern. Syst 50(11), 4415-4424 (2018)

14. Huang, J., Sun, L., Han, Z., Liu, L.: Adaptive terminal sliding mode control for nonlinear differential inclusion systems with disturbance. Nonlinear Dyn 72(1), 221-228 (2013)

15. Kim, K., Rew, K.: Reduced order disturbance observer for discrete-time linear systems. Automatica 49(4), 968-975 (2013)

16. Lin, S., Zhang, W.: Chattering reduced sliding mode control for a class of chaotic systems. Nonlinear Dyn 93(4), 2273-2282 (2018)

17. Lin, S., Zhang, W., Wang, H.: Controller designed via an adaptive reaching law for DSMC systems. IEEE Trans. Circuits Syst. II Express Briefs 67(2), 330-334 (2019)

18. Liu, J., Sun, M., Chen, Z., Sun, Q.: Super-twisting sliding mode control for aircraft at high angle of attack based on finite-time extended state observer. Nonlinear Dyn 99(4), 2785-2799 (2020)

19. Pai, M.C.: Discrete-time sliding mode control for robust tracking and model following of systems with state and input delays. Nonlinear Dyn 76(3), 1769-1779 (2014)

20. Roy, S., Baldi, S., Fridman, L.: On adaptive sliding mode control without a priori bounded uncertainty. Automatica 111, 108650 (2020)

21. Sharma, N., Janardhanan, S.: Discrete-time higher-order sliding mode control of systems with unmatched uncertainty. Int. J. Robust Nonlinear Control 29, 135-152 (2019)

22. Shtessel, Y., Edwards, C., Fridman, L., Levant, A.: Sliding Mode Control and Observation. Birkhäuser, Boston (2014)

23. Su, W., Drakunov, S., Ozguner, U.: An $O\left(T^{2}\right)$ boundary layer in sliding mode for sampled-data systems. IEEE Trans. Autom. Control 45(3), 482-485 (2000)

24. Sun, H., Guo, L.: Output regulation control for MIMO nonlinear system with mismatched disturbances and its application to BTT missiles. In: 11th World Congress on Intelligent Control and Automation, Shenyang, China, pp. 1130-1136 (2014)

25. That, N.D., Ha, Q.P.: Discrete-time sliding mode control with state bounding for linear systems with time-varying delay and unmatched disturbances. IET Control Theory Appl 9(11), 17001708 (2015)

26. Utkin, V.: Variable structure systems with sliding modes. IEEE Trans. Autom. Control 22(2), 212-222 (1977)

27. Veselić, B., Draženović, B., Milosavljević, Č.: Optimal discretetime integral sliding manifold design for linear systems subjected to a class of unmatched disturbances. In: International Workshop on Recent Advances in Sliding Modes, Istanbul, Turkey, pp. 1-6 (2015)

28. Wang, B., Yu, X., Chen, G.: ZOH discretization effect on singleinput sliding mode control systems with matched uncertainties. Automatica 45(1), 118-125 (2009)
29. Wang, J., Li, S., Yang, J., Wu, B., Li, Q.: Extended state observerbased sliding mode control for PWM-based DC-DC buck power converter systems with mismatched disturbances. IET Control Theory Appl 9(4), 579-586 (2015)

30. Wang, J., Wu, Y., Dong, X.: Recursive terminal sliding mode control for hypersonic flight vehicle with sliding mode disturbance observer. Nonlinear Dyn 81(3), 1489-1510 (2015)

31. Xia, Y., Zhu, Z., Li, C., Yang, H., Zhu, Q.: Robust adaptive sliding mode control for uncertain discrete-time systems with time delay. J. Frankl. Inst 347(1), 339-357 (2010)

32. Xu, Q.: Output-based discrete-time sliding mode control for a piezoelectrically actuated system. Nonlinear Dyn 76(1), 551-559 (2014)

33. Yang, J., Li, S., Yu, X.: Sliding-mode control for systems with mismatched uncertainties via a disturbance observer. IEEE Trans. Ind. Electron 60(1), 160-169 (2012)

34. Yang, Y., Guo, Z., Xiong, H., Ding, D.W., Yin, Y., Wunsch, D.C.: Data-driven robust control of discrete-time uncertain linear systems via off-policy reinforcement learning. IEEE Trans. Neural Netw. Learn. Syst. 30(12), 3735-3747 (2019)

35. Yao, J., Deng, W.: Active disturbance rejection adaptive control of uncertain nonlinear systems: Theory and application. Nonlinear Dyn 89(3), 1611-1624 (2017)

36. Zhang, J., Zhang, N., Shen, G., Xia, Y.: Analysis and design of chattering-free discrete-time sliding mode control. Int. J. Robust Nonlinear Control 29, 6572-6581 (2019) 